\title{
Fracture and determination of a lifetime of in- service mechanical components with porosity cluster in an aluminium alloy
}

\author{
Julien Vasseur ${ }^{1,1}$, Dmytro Vasiukov ${ }^{1}$, Salim Chaki ${ }^{1}$, Isabel Huther ${ }^{2}$, Michel Marzin ${ }^{2}$, \\ Fabien Lefebvre ${ }^{2}$, Benoît Dupont ${ }^{2}$, and Nicolas Leymarie ${ }^{3}$. \\ ${ }^{1}$ Institut Mines Télécom Lille-Douai, France \\ ${ }^{2}$ CETIM (Senlis) \\ ${ }^{3}$ CEA (Saclay)
}

\begin{abstract}
This paper focuses on the fatigue lifetime estimation of metallic components containing group of porosities as manufacturing defects. In order to represent these defects, six test specimens with different Side Drilled Holes (SDH) clusters, have been made of 7075-T6 aluminium alloy. Main characteristics of such defect (elementary size of porosities, spatial density or gradient of elementary sizes) have been studied by taking it into account in a FEM modelling in fatigue to determine their influence on the lifetime. Experimental tensile fatigue tests have been performed on all SDH samples with load ratio $\mathrm{R}=0.1$. Semi-elliptical surfaces with a size of a typical $\mathrm{Mg}_{2} \mathrm{Si}$ particle of the studied alloy have been chosen as initial cracks for multiple crack propagation. Indeed, such a particle is often responsible for the initiation of a crack in this type of material. For crack growth, Paris' type propagation law has been used. Local stress redistribution due to presence of the SDH implies mix-mode behaviour which has been considered by using G-theta method. Simulations results allowed realistic lifetime estimation according to the experimental ones. Moreover, a strong dependency of the crack paths and fatigue lifetime on the defect characteristics has been proved and quantified.
\end{abstract}




\section{Introduction}

In the literature, we can find amounts of examples dealing with the influence of porosity on the lifetime of structures in aluminium [1-3]. For geometrically complex cases, the porosity is often considered as an equivalent defect, which has the equivalent "Murakami size" [4]. In order to simulate a porosity cluster, and to master all its geometrical parameters, we have chosen to machine some SDH in plate samples. We studied the influence of different configurations of SDH in the aluminium 7075-T6 on the fatigue lifetime. These configurations are used to test the main characteristics of porosity cluster one at a time. We studied the influence on fatigue of some characteristics of porosity as the elementary size of porosities, their spatial density or the gradient in their elementary sizes. In the present paper, we only illustrate a case where the SDH are very small $(150 \mu \mathrm{m}$ of diameter $)$ and close $(0.5 \mathrm{~mm}$ between two nearby SDH) to each other. Currently, lifetime estimations are often very conservative, because they are made according to an equivalent defect which encompasses the porosity. This paper focuses on the simulation of the fatigue crack propagation along the $\mathrm{SDH}$, to improve fatigue life prediction of structures containing porosity. Fatigue load $\left(\mathrm{R}=0.1, \mathrm{~F}_{\max }=13.5 \mathrm{kN}\right)$ is applied on the samples and considered sets of SDH imply multi-crack initiations and local mixed-mode behaviour while the crack propagation. In order to take into account this phenomenon, numerical simulations which employ the G-theta method, described in [5], are provided. For this paper, we first describe the material and the protocol and we report the experimental fatigue propagation crack results. Then the experimental fracture surfaces are used to determine input parameters for the numerical simulation of the phenomenon. Eventually, we compare the results between the two lifetimes obtained.

\section{Description of specimens}

In this study, the considered material is the aluminium 7075-T6. Considering the classic Paris law:

$$
\frac{d a}{d N}=C(\Delta K)^{m}
$$

We consider the mechanical parameters from the literature survey [6] described in the Table 1 .

Table 1. Mechanical parameters of the classical Paris law

\begin{tabular}{|c|c|}
\hline$E$ & $71 \mathrm{MPa}$ \\
\hline $\mathrm{C}$ & $9.83 .10^{-14}$ \\
\hline $\mathrm{m}$ & 3.64 \\
\hline
\end{tabular}

\section{Geometry}

The samples are cut in a rolled sheet and are dog-bone shape, with a thickness of $3 \mathrm{~mm}$. The central part is $30 \mathrm{~mm}$ width and $60 \mathrm{~mm}$ length. SDH are placed at the centre of the sample. 

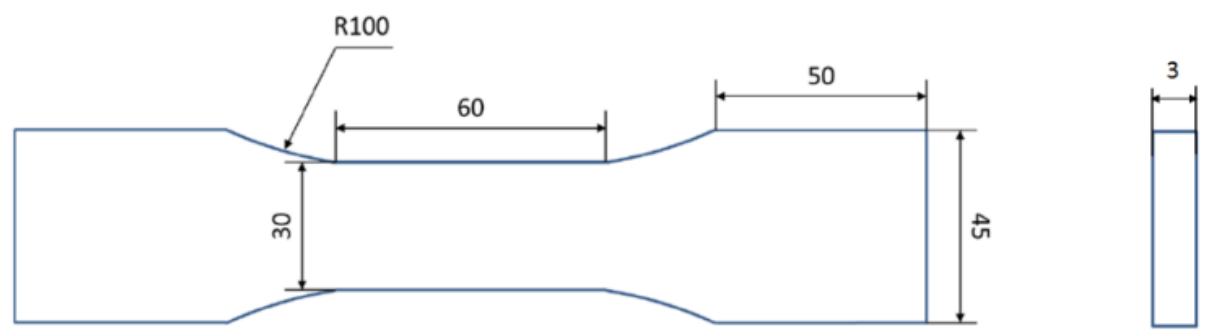

Fig. 1. Dimensions of the samples in $\mathrm{mm}$

The SDH inside are positioned and dimensioned to simulate the principle characteristics of a group of porosities. The presented configuration is noted "elementary case". The size of the $7 \mathrm{SDH}$ is very small $(150 \mu \mathrm{m}$ diameter, which is in the order of micro porosity). They are disposed like a hexagon which is $15^{\circ}$-rotated with the loading axis, to avoid particular alignments. For comparison, a classical equivalent defect for calculations, noted "envelope case", composed of a unique defect which size is the global size of defects of the previous case cluster ( $2 \mathrm{~mm}$ diameter) was studied. Both configurations are shown in Fig. 2.
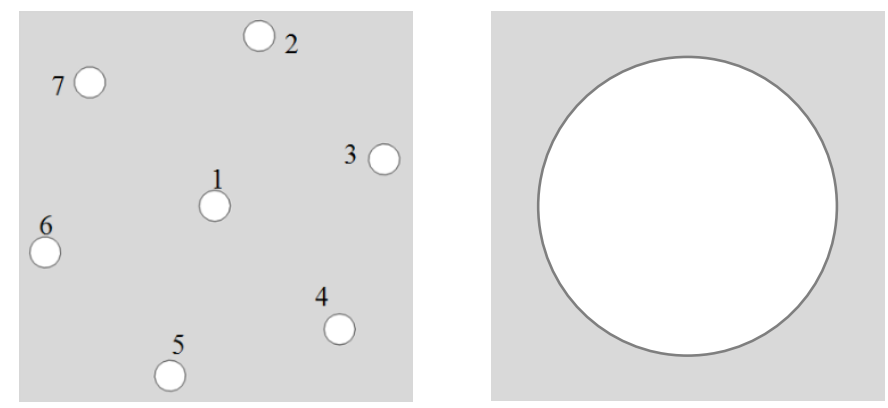

Fig. 2. Illustration of the defects positions. Elementary case (left); Envelope case (right), which is classically considered as the equivalent defect;

\section{Loading}

The fatigue load is tension with $\mathrm{R}=0.1$. The maximum load is set to $13.5 \mathrm{kN}$, which is equivalent to $150 \mathrm{MPa}$ in the central part of the sample. Some tests were carried out at $10 \mathrm{~Hz}$, or $15 \mathrm{~Hz}$ (see Fig. 3).

\section{Experimental results}

First, in order to better characterize our aluminium in fatigue, we made some tests on samples without defect (sane samples) to fit an S-N curve. Then we carried out some tests with defects: "envelope case" and "elementary case". The experimental results are shown in Fig. 3. 


\section{Smax-Nf curve $(\mathbf{R}=0,1)$}

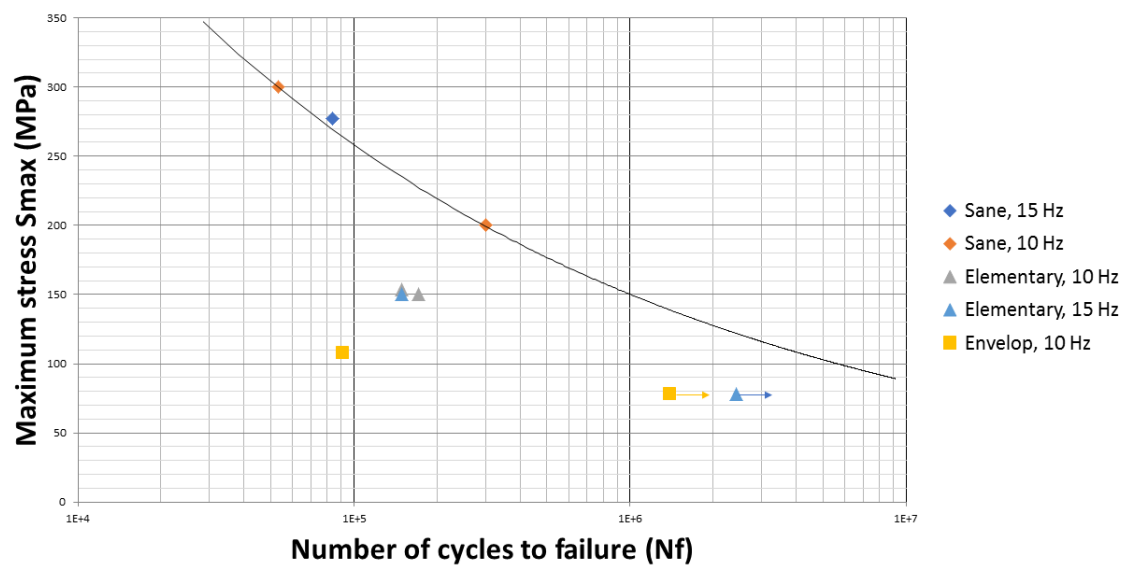

Fig. 3. Smax-Nf curve $(\mathrm{R}=0.1)$

Fig. 3 shows that the "envelop case" fatigue strength is lower than the "elementary case". The "elementary case" samples were failed after 148770 cycles for the first test and 171804 cycles for the second. Some binocular observations are shown below:
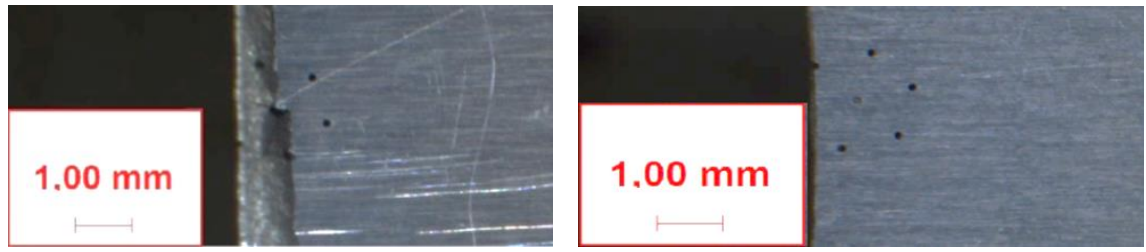

Fig. 4. Profile views (elementary case, two faces)

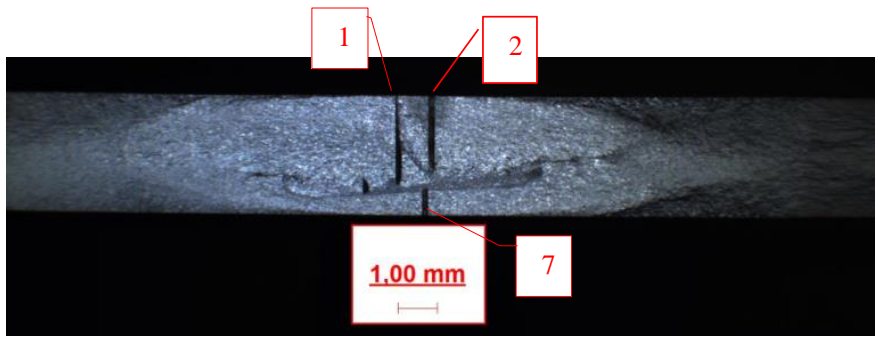

Fig. 5. Top view (elementary case)

We can observe that the crack passes through the central defect on the Fig. 4 and that a stair is clearly visible on Fig. 5, which suggests that several cracks propagate from multiple locations. Nevertheless, the comparison of the sizes of these fatigue zone steps shows that the greatest crack contains the first initiation zone (those at the left on Fig. 5). The distinction between fatigue propagation and sudden break is clearly visible on Fig. 5.The crack propagation is approximately symmetrical to the left and the right of the defect $\mathrm{n}^{\circ} 1$ and the crack measures approximately $13 \mathrm{~mm}$. The cracks are orthogonal to the tensile direction. 


\section{Static simulation}

At the first step, a static calculation has been performed to determine the localization of the initiation of a defect. The mesh size is small enough to have approximately 100 elements around each SDH. Elements used are CPS4R.
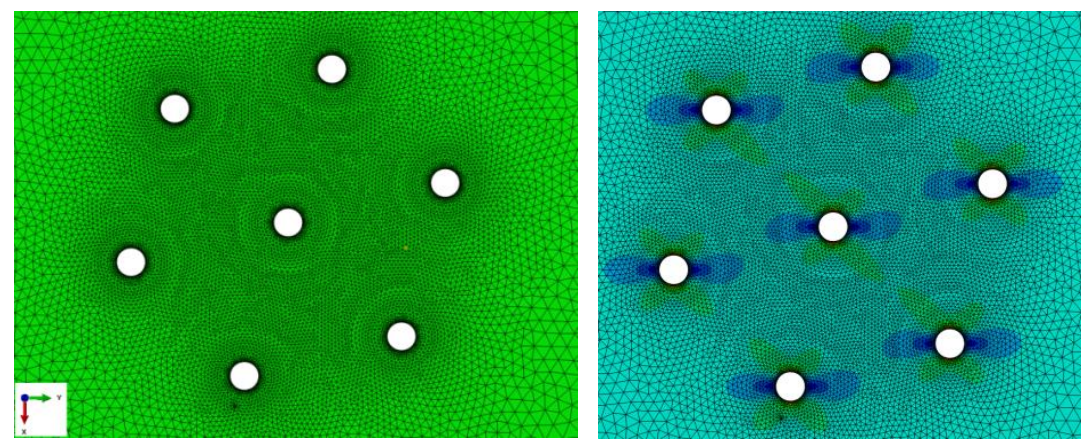

Fig. 6. Illustration of the mesh (tension along y axis)

The criterion chosen is the maximum stress equivalent of Von Mises. According to the simulations, the crack initiation seems to be approximately equiprobable from all the defects. To fatigue propagation simulation, we decided to put an elliptical initial crack on the central defect, along the thickness, according to some fracture surface experimentally obtained.

\section{Fatigue simulation methodology}

In this part, we aim at reproducing the experiments that we did. The phenomenon that we observed allowed us to make some hypothesis to simulate it. In order to have a numerical prediction of the lifetime of the sample, the software which was used is Zcracks. It allowed us to propagate an initial crack which is inserted in a sane model meshed on Abaqus.

The used crack growth algorithm is restrained to the case of fatigue loading. The Paris' law is used as crack propagation law. During computation, the energy release rate $G$ is computed using the G-Theta method [5]. The particularity of the method is to consider a crack front discretized with $\mathrm{n}_{\mathrm{p}}$ nodes (or points) so that a curvilinear coordinate system can be defined. The number of control points of the crack front $\left(n_{p}\right)$ is chosen by the user. In linear elasticity, the energy release rate verifies the variational equation:

$$
\int_{\Gamma_{0}} \mathrm{G}(\mathrm{s}) \cdot \boldsymbol{\theta}(\mathrm{s}) \cdot \mathbf{n}(\mathrm{s}) \cdot \mathrm{ds}=J(\boldsymbol{\theta})
$$

Where $\Gamma_{0}$ is the crack front. $G$ and $\boldsymbol{\theta}$ are then discretized in shape functions $\mathrm{N}_{\mathrm{i}}$, which gives:

$$
\sum_{j=1}^{n_{p}} G_{j} \cdot \int_{\Gamma_{0}} N_{\mathrm{i}}(s) \cdot N_{\mathrm{j}}(s) \cdot d s=J\left(\boldsymbol{\theta}^{i}\right)
$$

Where $i$ is a point on the crack front, and $\mathrm{J}$ calculated as follows:

$$
J\left(\boldsymbol{\theta}^{i}\right)=\iiint_{\Omega}\left[\frac{1}{2}(\overline{\bar{\sigma}}: \overline{\bar{\varepsilon}}) \cdot \operatorname{div}\left(\theta^{i}\right)-\overline{\bar{\sigma}}:\left(\overrightarrow{\operatorname{grad} u} \cdot \overrightarrow{\operatorname{grad}} \theta^{i}\right)\right] d \Omega
$$

Where $\mathrm{N}_{\mathrm{j}}$ are shape functions, and $\theta^{\mathrm{i}}$ are virtual crack extension velocity fields verifying:

$$
\theta^{\mathrm{i}}(s) \cdot e_{1}(s)=N_{i}(s)
$$


Westergaard displacement solutions $v^{I, I I, I I I}$ defined in the crack front vicinity, allows to compute associated $G^{v, I, I I, I I I}$ values. The following Irwin formula for a given isotropic linear elastic behaviour, leads to each associated SIF $K_{j}^{I, I I, I I I}$ along the front discretization:

$$
\sum_{j=1}^{n_{p}} G_{j}^{I, I I, I I I} \cdot \int_{\Gamma_{0}} N_{\mathrm{i}}(s) \cdot N_{\mathrm{j}}(s) \cdot d s=\sum_{j=1}^{n_{p}} \frac{1-v^{2}}{E}\left(K_{j}^{I} \cdot K_{j}^{v, I}+K_{j}^{I I} \cdot K_{j}^{v, I I}\right)+\frac{1}{2 v} K_{j}^{I I I} \cdot K_{j}^{v, I I I} \int_{\Gamma_{0}} N_{\mathrm{i}}(s) \cdot N_{\mathrm{j}}(s) \cdot d s
$$

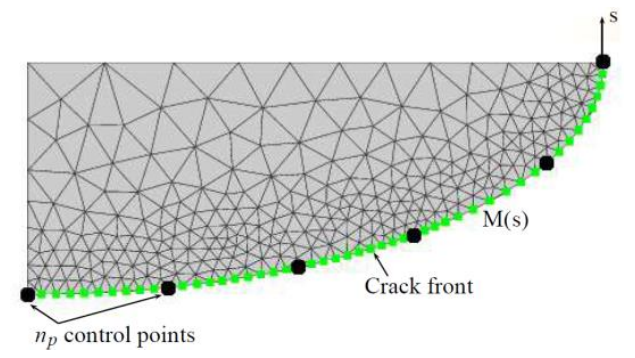

Fig. 7. Illustration of the crack front [7]

The algorithm can be described as follows [6]:

1. Initialization: $K_{\max }=K_{\min }=0$;

2. $\Delta K$ is computed accordingly to the G-theta method;

3. Compute current time step and keep extreme $K$ values for the current cycle;

4. Compute $d a$ for every node along the crack front;

5. Get maximal $d a$ value. If it is greater than a specified $d a_{\max }$ parameter controlling crack growth, compute $d N$ accordingly for Paris law integration;

6. $\quad$ Update $N$ with $d N$ value and process remeshing with incremented crack position;

7. Go back to 1;

\section{Model description for fatigue simulations}

For simplicity, only one initial crack is placed orthogonally to the direction of loading on the SDH central defect. The initial crack represents an ellipse $(10 \mathrm{~mm} \times 0.21 \mathrm{~mm})$ to have a crack approximately rectangular along the depth and cuts the geometry at the central SDH (Fig. 8). Its depth is taken equal to $30 \mu \mathrm{m}$ on either sides of the hole because it is the order of size of $\mathrm{Mgg}_{2} \mathrm{Si}$ and $\mathrm{Cu}_{2} \mathrm{Fe}$ particles, which are often responsible for the fatigue crack initiation in the aluminium alloys $7 \mathrm{xxx}[8,9]$. These authors add that majority $(60-75 \%)$ of these particles have generally initial crack at the initial state in the case of a rolled sheet (as present). It is the reason why we will neglect here the crack initiation time. Initial crack is placed orthogonally to the direction of loading. The mesh is locally $0.0125 \mathrm{~mm}$ size at least (i.e. approximately 40 times smaller than the perimeter of each defect) and has a maximum size of $3 \mathrm{~mm}$ with a gradation factor of 1.3 when distance to the crack front is growing. The crack growth rate is supposed to follow the Paris' law which coefficients are detailed in Table 1. The simulation is considered finished when the crack tip is at least $6.5 \mathrm{~mm}$ distant from the centre of the sample, corresponding to the dimensions observed on the tested specimen fractures. The energy release rate $\mathrm{G}$ is computed every 8 nodes on the crack front. The mesh is quadratic and the elements used are C3D10. At each increment a region of 0.3 $\mathrm{mm}$ around the crack tip is re-meshed because it is 2 times the size of a SDH. The angle of bifurcation is computed by the maximum- $\sigma_{\theta \theta}$ criterion. 

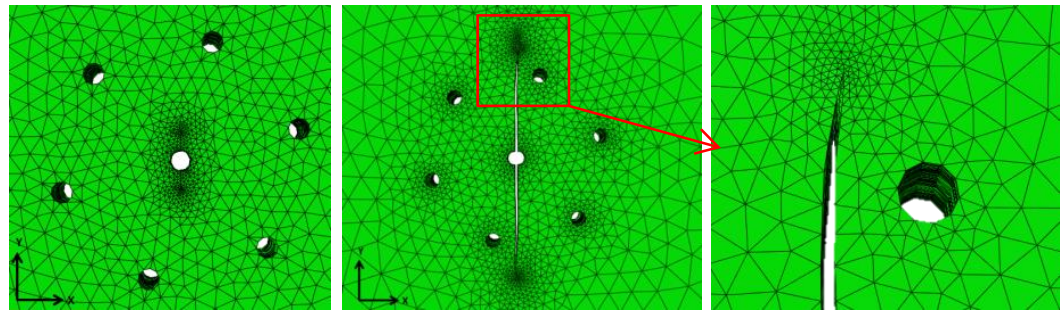

Fig. 8. Illustration of the crack propagation and zoom on the top tip;

\section{Simulation results}

According to the simulation, the number of cycles for the crack to quit the SDH cluster is 197781 . For the end of propagation, it can easily be solved analytically. Considering the same Paris law and the same initial/final crack criteria, we find 30666 cycles. The total number of cycles is then 228447 .

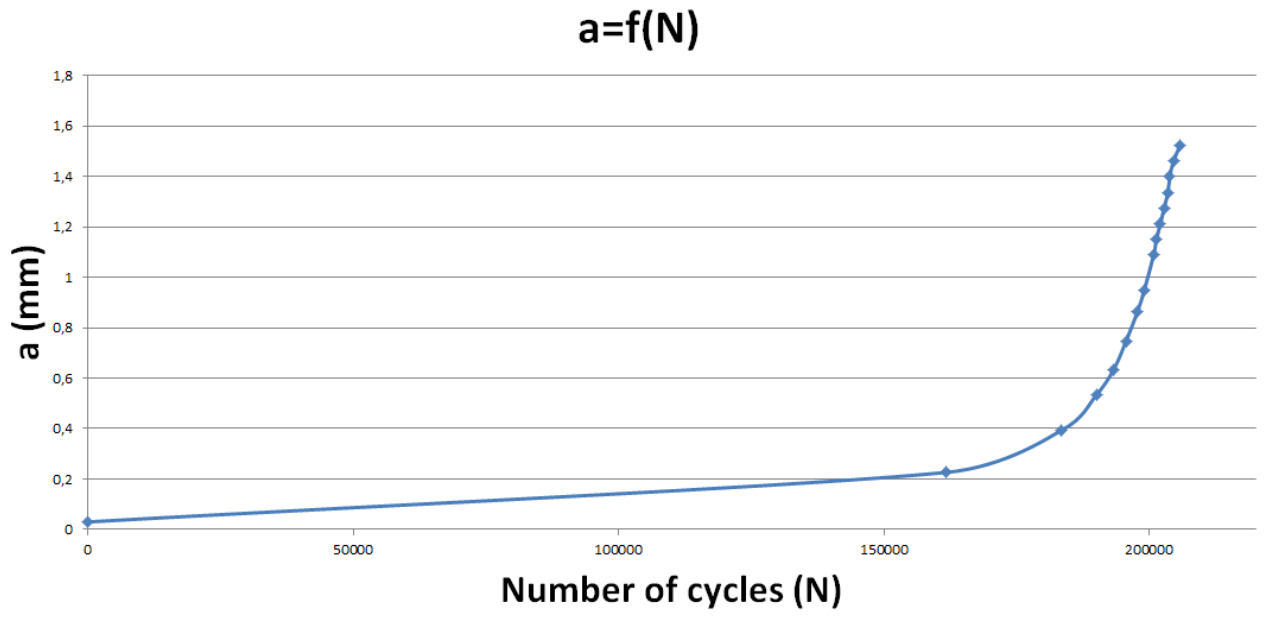

Fig. 9. $a=f(N)$ curve

Experimentally, the propagation in mode I seems to be preponderant when a unique crack initiate from the central SDH. But sometimes we observe multi-initiations of fatigue cracks, which coalesce in a mixed-mode behavior and finally propagate essentially in mode I before the sudden break as in Fig. 5.

\section{Conclusion}

Currently, the studied case is not easy to treat, because multi-mode behavior is a priori present and no standardization exists for the treated case. Furthermore, crack initiation is difficult to foresee, because the defects are all close enough to possibly initiate a crack. 
The simulated cracks path matches correctly some experimental fracture surfaces. Others experimental fracture surfaces are different, cause of a crack multi-initiation occurs. In such a case, approximations with an envelop equivalent defect are too conservative. Our calculation of the lifetime gave a correct, but non conservative estimation. It can be explained by the sudden break zone, considered to be at $13 \mathrm{~mm}$. Indeed, it is not exactly the case, because the fatigue zone is oval, due to side effects. It could be improved by using other propagation laws, taking into account the effect of short cracks (at the beginning of the phenomenon) or the crack-closure effect. Some simulations are running to model a multiple crack initiation to obtain the same fracture surface as those shown in Fig. 5.

\section{References}

1. E. Charkaluk, A. Constantinescu, F. Szmytka, S. Tabibian. Probability density functions: From porosities to fatigue lifetime. International Journal of Fatigue (2014).

2. Y.X. Gao, J.Z. Yi., P.D. Lee, T.C. Lindley. The effect of porosity on the fatigue life of cast aluminium-silicon alloys. Fatigue and Fracture of Engineering Materials and Structures. (2004).

3. V. Bonnand, S.Pierret, V.Chiaruttini, E. Andrieu, \& E. Fessler. Relation between crack growth behaviour and crack front morphology under hold-time conditions in DA Inconel 718. International Journal of Fatigue, 17-27, (2016).

4. Y. Murakami, K. Kawakami, \& W.E. Duckworth. Quantitative Evaluation of Effects of Size and Shape of Artificially Introduced Alumina Inclusions on the Fatigue Strength of Ni-Cr-Mo Steel. Tetsu-to-Hagane. (2017).

5. S. Geniaut, P. Massin, N. Moes. Evaluation of stress intensity factors with g-theta method and level sets in code aster.

6. J. Melson, J.Kennedy. Fatigue Crack Growth Analysis with Finite Element Methods and a Monte Carlo Simulation, Thesis (2014).

7. Z-crack tutorial: http://www.zset-software.com/support/manuals/.

8. Y. Xue, H. El Kadiri, M.F. Horstemeyer, J.B. Jordon, H. Weiland. Micromechanisms of multistage fatigue crack growth in a high-strength aluminum alloy. Acta Materialia, 55(6), 1975-1984 (2007).

9. W.L. Morris, The effect of intermetallics composition and microstructure on fatigue crack initiation in Al 2219-T851. Metallurgical and Materials Transactions A, 9A: 1345-1348 (1978). 